Stud. Univ. Babeş-Bolyai Math. 64(2019), No. 2, 279-289

DOI: 10.24193/subbmath.2019.2.12

\title{
Parameter estimations for linear parabolic fractional SPDEs with jumps
}

\author{
Wilfried Grecksch, Hannelore Lisei and Jens Lueddeckens
}

\begin{abstract}
We give an unbiased and consistent estimator for the drift coefficient of a linear parabolic stochastic partial differential equation driven by a multiplicative cylindrical fractional Brownian motion with Hurst index $1 / 2<h<1$ and a cylindrical centered Poisson process, if the observations of the solution process are given in discrete time points. The presented method is based on mean square estimations.
\end{abstract}

Mathematics Subject Classification (2010): 60H15, 62F12, 60G22.

Keywords: Parameter estimation, SPDE, cylindrical fractional Brownian motion, cylindrical Poisson process.

\section{Introduction}

There are lots of papers concerning parameter estimations for stochastic differential equations (SDEs). Results for SDEs driven by a fractional Brownian motion (fBm) were given, for example, by Y. Kozachenko, A. Melnikov, Y. Mishura [4] and W.L. Xiao, W.G. Zhang, X. Zhang [11] (see also the references therein). If the driving process is a Lévy process, then see, for example, H. Long [5] and the references therein. If the equations are driven by a $\mathrm{fBm}$ and a fractional Poisson measure one can find interesting results and applications in the $\mathrm{PhD}$ thesis of J. Lueddeckens [7].

Many papers are devoted to the parameter estimation of fractional stochastic partial differential equations (SPDEs). As a representative result we quote here the paper [8] of B. Maslowski and C.A. Tudor.

The following estimation criteria are mainly used in constructing estimators for the parameters of SPDEs:

- maximum likelihood type methods by considering fundamental martingales and theorems of Girsanov type;

This paper has been presented at the fourth edition of the International Conference on Numerical Analysis and Approximation Theory (NAAT 2018), Cluj-Napoca, Romania, September 6-9, 2018. 
- time continuous and time discrete least square criteria;

- Kalman-Bucy filters;

- $L^{1}$-norm estimations and contrast estimations.

Often SPDEs are considered as stochastic evolution equations in Hilbert spaces. For example, a parameter estimation problem for linear diagonalized stochastic partial differential equations driven by a multiplicative fBm is considered by I. Cialenco in [2]. The stochastic processes defined by the random Fourier coefficients of the solution process describe one dimensional geometric fractional Brownian motions. Based on these processes, consistent parameter estimates for the SPDEs are determined using a maximum likelihood type method. So we see, that one dimensional results of parameter estimations are useful for parameter estimations of SPDEs.

Parameter estimations for diagonal SPDEs are also considered in Chapter 6 in [6] by S.V. Lototsky, B.L. Rozovsky.

The aim of the present paper is to give new contributions in the estimation theory of the coefficients of linear homogeneous SPDEs, which are driven by cylindrical fractional Brownian motions and cylindrical Poisson processes. The applied estimation criterion uses covariances (as a generalization of the mean squared method), such that the long range dependence property of the fractional Brownian motion with Hurst index $h \in] 1 / 2,1[$ is taken into account. Moreover, in this paper weakly, respectively strongly consistent estimators are constructed by using only information about the underlying process in discrete time points.

The paper starts with a preliminary section, containing the assumptions needed throughout the paper. A linear SPDE driven by a multiplicative cylindrical fractional Brownian motion and a cylindrical Poisson process is introduced in Section 3. In Section 4 the one dimensional stochastic differential equations for the Fourier coefficients of the solution process of the SPDE is considered and similar to the results from [7] an estimation criterion of least squares type in discrete time points of the observations for the drift term is formulated. The estimator of the drift coefficient is unbiased. Conditions for choosing the time points are given such that the constructed estimator is unbiased and weakly consistent (Theorem 4.2), respectively strongly consistent (see Theorem 4.4).

\section{Preliminaries}

Definition 2.1. A real-valued Gaussian process $\left(B^{h}(t)\right)_{t \geq 0}$ with $E\left(B^{h}(t)\right)=0$, for all $t \geq 0, B^{h}(0)=0$ and Hurst index $\left.h \in\right] 0,1[$ is called fractional Brownian motion (fBm) if

$$
E\left(B^{h}(t) B^{h}(s)\right)=\frac{1}{2}\left(t^{2 h}+s^{2 h}-|t-s|^{2 h}\right) \text { for all } s, t \geq 0 .
$$

The $\mathrm{fBm}$ is not a semimartingale and it is not a Markovian process for $h \neq 1 / 2$. The $\mathrm{fBm}$ is a Wiener process for $h=1 / 2$. In this paper we consider $h \in] 1 / 2,1[$. Then, the $\mathrm{fBm}$ has the so-called long range dependence property. 


\section{Assumptions:}

(A1) Let $\left(V, H, V^{*}\right)$ be a triplet of rigged Hilbert spaces, where $V$ is compactly embedded into $H$ and $A: V \rightarrow V^{*}$ is linear and $\langle A v, v\rangle+\alpha_{1}\|v\|_{V}^{2} \leq \alpha_{2}\|v\|_{H}^{2}$ for all $v \in V$ and $\alpha_{1}>0, \alpha_{2} \in \mathbb{R}$ are constants.

Observe, that $A: D(A) \rightarrow H$ is linear and unbounded with $D(A)=\{v \in V$ : $A v \in H\}$, which is dense in $H$. The eigenvalues $\left(\lambda_{k}\right)_{k \geq 1}$ of this operator are negative and satisfy $\lim _{k \rightarrow \infty} \lambda_{k}=-\infty$.

(A2) Let $\left(h_{k}\right)_{k \geq 1} \subset H$ be the complete orthonormal system constructed by the eigenfunctions of $A$.

(A3) The $C_{0}$ semigroup $\left(\mathcal{T}_{t}\right)_{t \geq 0}$ defined by

$$
\mathcal{T}_{t}(x)=\sum_{k=1}^{\infty} \exp \left\{\lambda_{k} t\right\}\left(x, h_{k}\right) h_{k}, x \in H
$$

is generated by $-A$, where $(\cdot, \cdot)$ denotes the scalar product in $H$.

(A4) $\Phi_{1}, \Phi_{2}: H \rightarrow H$ are Hilbert-Schmidt operators of the type

$$
\Phi_{i}(x)=\sum_{k=1}^{\infty} \mu_{i k}\left(x, h_{k}\right) h_{k}, \quad x \in H,
$$

where $\sum_{k=1}^{\infty} \mu_{i k}^{2}<\infty$ for $i \in\{1,2\}$.

(A5) Let $\left(B_{k}^{h}(t)\right)_{t \geq 0}, k \in\{1,2, \ldots\}$, be independent fractional Brownian motions with Hurst index $h \in] 1 / 2,1[$ and let

$$
B^{h}(t)=\sum_{k=1}^{\infty} B_{k}^{h}(t) h_{k}, \quad t \geq 0,
$$

denote the cylindrical $\mathrm{fBm}$.

(A6) Let $\left(\pi_{j}(t)\right)_{t \geq 0}, j \in\{1,2, \ldots\}$, be independent homogeneous Poisson processes with parameter $\nu$.

(A7) Consider $\tilde{\pi}_{j}(t)=\pi_{j}(t)-\nu t, j \in\{1,2, \ldots\}$ and we denote by

$$
\tilde{\pi}(t)=\sum_{j=1}^{\infty} \tilde{\pi}_{j}(t) h_{j}, \quad t \geq 0,
$$

the cylindrical centered Poisson process.

(A8) The processes $B_{k}^{h}$ and $\tilde{\pi}_{j}$ are independent stochastic processes for all $j, k \in$ $\{1,2, \ldots\}$.

(A9) Let $X_{0} \in H$ be a deterministic initial value.

(A10) All stochastic processes are defined on the same complete filtered probability space $\left(\Omega, \mathcal{F},\left(\mathcal{F}_{t}\right)_{t \geq 0}, P\right)$, where $\mathcal{F}_{t}=\sigma\left(\mathcal{F}_{t}^{B^{h}} \vee \mathcal{F}_{t}^{\tilde{\pi}}\right)$ and $\mathcal{F}_{t}^{B^{h}}$ and $\mathcal{F}_{t}^{\tilde{\pi}}$ denote the $\sigma$-algebras generated by $\left(B^{h}(s)\right)_{s \in[0, t[}$ and $(\tilde{\pi}(s))_{s \in[0, t[}$. 


\section{A linear fractional parabolic SPDE with jumps}

At first we introduce for $k \in\{1,2, \ldots\}$ the one dimensional linear stochastic differential equations

$$
d Y_{k}(t)=a \lambda_{k} Y_{k}(t) d t+\sigma \mu_{1 k} Y_{k}(t) d B_{k}^{h}(t)+\eta \mu_{2 k} Y_{k}(t-) d \tilde{\pi}_{k}(t),
$$

where $a, \sigma, \eta$ are positive constants and $Y_{k}(0)=\left(X_{0}, h_{k}\right)$.

The stochastic equation (3.1) is defined by

$$
Y_{k}(t)=Y_{k}(0)+a \lambda_{k} \int_{0}^{t} Y_{k}(s) d s+\sigma \mu_{1 k} \int_{0}^{t} Y_{k}(s) d B_{k}^{h}(s)+\eta \mu_{2 k} \int_{0}^{t} Y_{k}(s-) d \tilde{\pi}_{k}(s),
$$

for all $t \geq 0$, where the stochastic integral with respect to $B_{k}^{h}$ is defined by a divergence integral as in [9] and the stochastic integral with respect to the compensated Poisson process is defined as in [10], Chapter II (or [3], page 246).

Theorem 3.1. The process

$$
Y_{k}(t)=Y_{k}(0)\left(1+\eta \mu_{2 k}\right)^{\pi_{k}(t)} \cdot \exp \left\{\sigma \mu_{1 k} B_{k}^{h}(t)-\frac{1}{2} \sigma^{2} \mu_{1 k}^{2} t^{2 h}+a \lambda_{k} t-\nu \eta \mu_{2 k} t\right\}
$$

solves equation (3.1) for all $t \geq 0$ with probability 1 .

Proof. We prove that the process $Y_{k}(t)=Y_{1 k}(t) Y_{2 k}(t)$ with

$$
Y_{1 k}(t)=\exp \left\{\sigma \mu_{1 k} B_{k}^{h}(t)-\frac{1}{2} \sigma^{2} \mu_{1 k}^{2} t^{2 h}\right\}
$$

and

$$
Y_{2 k}(t)=Y_{k}(0)\left(1+\eta \mu_{2 k}\right)^{\pi_{k}(t)} \cdot \exp \left\{a \lambda_{k} t-\nu \eta \mu_{2 k} t\right\}
$$

is the solution of (3.1). Since the fBms and the Poisson processes are independent, we get

Obviously it holds

$$
d Y_{k}(t)=Y_{1 k}(t) d Y_{2 k}(t)+Y_{2 k}(t) d Y_{1 k}(t)
$$

$$
Y_{2 k}(t)=Y_{k}(0) \exp \left\{a \lambda_{k} t-\nu \eta \mu_{2 k} t+\pi_{k}(t) \ln \left(1+\eta \mu_{2 k}\right)\right\} .
$$

It follows by a result from [3] (see formula (15) on page 261) that (3.5) solves

$$
d Y_{2 k}(t)=Y_{2 k}(t)\left[a \lambda_{k}-\nu \eta \mu_{2 k}\right] d t+\eta \mu_{2 k} Y_{2 k}(t-) d \pi_{k}(t)
$$

with $Y_{2 k}(0)=Y_{k}(0)$. By the definition of $\tilde{\pi}(t)$ it follows

$$
d Y_{2 k}(t)=a \lambda_{k} Y_{2 k}(t) d t+\eta \mu_{2 k} Y_{2 k}(t-) d \tilde{\pi}_{k}(t)
$$

with $Y_{2 k}(0)=Y_{k}(0)$.

The fractional Itô formula in [9] (see formula (2.18)) gives that the process $Y_{1 k}(t)$ solves

with $Y_{1 k}(0)=1$.

$$
d Y_{1 k}(t)=\mu_{1 k} \sigma Y_{1 k}(t) d B_{k}^{h}(t)
$$

If we substitute these results in (3.4), then we get that the process (3.3) solves equation (3.1).

We will prove the following a priori estimates: 
Lemma 3.2. There are constants $C_{0}>0, C_{1}>0$ and $C_{2}>1$ such that for all $t \geq 0$ and $k \in\{1,2, \ldots\}$ it holds

$$
E\left|Y_{k}(t)\right|^{2} \leq F(t)\left|Y_{k}(0)\right|^{2}
$$

with

$$
F(t)=C_{0} \exp \left\{2 \sigma^{2} C_{1} t^{2 h}+\nu t\left(C_{2}-1\right)\right\} .
$$

Proof. Since the fBms and the Poisson processes are independent, we get

$$
\begin{aligned}
E\left|Y_{k}(t)\right|^{2}= & \left|Y_{k}(0)\right|^{2}\left(E \exp \left\{2 \sigma \mu_{1 k} B_{k}^{h}(t)\right\}\right)\left(E\left(\left(1+\eta \mu_{2 k}\right)^{2 \pi_{k}(t)}\right)\right) \\
& \times \exp \left\{-\sigma^{2} \mu_{1 k}^{2} t^{2 h}+2 a \lambda_{k} t-2 \nu \eta \mu_{2 k} t\right\} .
\end{aligned}
$$

Since $\lambda_{k}<0$ for each $k \in\{1,2, .$.$\} and \left(\mu_{2 k}\right)_{k \geq 1}$ is a bounded sequence, we have

$$
\exp \left\{-\sigma^{2} \mu_{1 k}^{2} t^{2 h}+2 a \lambda_{k} t-2 \nu \eta \mu_{2 k} t\right\} \leq C_{0} \text { for each } t \geq 0,
$$

where $C_{0}>0$ is a constant.

The random variable $Z_{1}:=\exp \left\{2 \sigma \mu_{1 k} B_{k}^{h}(t)\right\}$ is log-normally distributed, so we get for its expectation

$$
E\left(Z_{1}\right)=\exp \left\{2 \sigma^{2} \mu_{1 k}^{2} t^{2 h}\right\} .
$$

From the boundedness of $\left(\mu_{1 k}\right)_{k \geq 1}$ it follows the existence of a positive constant $C_{1}$ with

$$
E\left(Z_{1}\right) \leq \exp \left\{2 \sigma^{2} C_{1} t^{2 h}\right\} .
$$

For $Z_{2}:=\left(1+\eta \mu_{2 k}\right)^{2 \pi_{k}(t)}$ we compute

$$
E\left(Z_{2}\right)=E\left(\left(1+\eta \mu_{2 k}\right)^{2 \pi_{k}(t)}\right)=\sum_{j=0}^{\infty}\left(1+\eta \mu_{2 k}\right)^{2 j} \exp \{-\nu t\} \frac{(\nu t)^{j}}{j !}
$$

But the sequence $\left(\mu_{2 k}^{2}\right)_{k \geq 1}$ is bounded, hence there is a constant $C_{2}>1$ such that

$$
E\left(Z_{2}\right) \leq \exp \{-\nu t\} \sum_{j=0}^{\infty} \frac{\left(\nu t C_{2}\right)^{j}}{j !}=\exp \left\{\nu t\left(C_{2}-1\right)\right\} .
$$

Then we get with (3.7) the inequality (3.6).

We now consider a solution definition of mild solution type.

Theorem 3.3. Let $k \in\{1,2, \ldots\}$. The process $\left(Y_{k}(t)\right)_{t \geq 0}$ defined by (3.3) solves (3.2) if and only if the equation

$$
\begin{aligned}
Y_{k}(t) & =Y_{k}(0) \exp \left\{\lambda_{k} a t\right\}+\int_{0}^{t} \exp \left\{\lambda_{k} a(t-s)\right\} \sigma \mu_{1 k} Y_{k}(s) d B_{k}^{h}(s) \\
& +\int_{0}^{t} \exp \left\{\lambda_{k} a(t-s)\right\} \eta \mu_{2 k} Y_{k}(s-) d \tilde{\pi}_{k}(s), \text { for all } t \geq 0
\end{aligned}
$$

holds. 
Proof. If the process $\left(Y_{k}(t)\right)_{t \geq 0}$ is the solution of (3.2) defined by (3.3), then this process solves (3.8) too.

Let $\left(\tilde{Y}_{k}(t)\right)_{t \geq 0}$ be a solution process of $(3.8)$. Then we get

$$
\begin{gathered}
\tilde{Y}_{k}(t)=Y_{k}(0) \exp \left\{a \lambda_{k} t\right\}+\exp \left\{a \lambda_{k} t\right\} \int_{0}^{t} \exp \left\{-a \lambda_{k} s\right\} \sigma \mu_{1 k} \tilde{Y}_{k}(s) d B_{k}^{h}(s) \\
+\exp \left\{a \lambda_{k} t\right\} \int_{0}^{t} \exp \left\{-a \lambda_{k} s\right\} \eta \mu_{2 k} \tilde{Y}_{k}(s-) d \tilde{\pi}_{k}(s) .
\end{gathered}
$$

Obviously $\exp \left\{a \lambda_{k} t\right\}$ is deterministic and differentiable and the stochastic differentials of the stochastic integrals in formula (3.9) exist. If we use the stochastic product formula to the two last terms of the sum in formula (3.9), then we get

$$
d \tilde{Y}_{k}(t)=a \lambda_{k} \tilde{Y}_{k}(t) d t+\sigma \mu_{1 k} \tilde{Y}_{k}(t) d B_{k}^{h}(t)+\eta \mu_{2 k} \tilde{Y}_{k}(t-) d \tilde{\pi}_{k}(t) .
$$

It follows from formula $(3.9)$ that $\tilde{Y}_{k}(0)=Y_{k}(0)$. That is, $\tilde{Y}_{k}(t)=Y_{k}(t)$ for all $t \geq 0$ with probability 1.

We introduce for $n \geq 1$ and $t \geq 0$

$$
X_{n}(t):=\sum_{k=1}^{n} Y_{k}(t) h_{k}
$$

By $(3.6)$ and $Y_{k}(0)=\left(X_{0}, h_{k}\right)$ we get

$$
E\left\|X_{n}(t)\right\|_{H}^{2}=\sum_{k=1}^{n} E\left|Y_{k}(t)\right|^{2} \leq\left\|X_{0}\right\|_{H}^{2} F(t)
$$

for every $t>0$. It follows also from this inequality and the definition of $F(t)$ that there is for all $T>0$ a positive constant $C_{T}$ such that

$$
E\left\|X_{n}(t)\right\|_{H}^{2} \leq C_{T}\left\|X_{0}\right\|_{H}^{2}
$$

and

$$
E \int_{0}^{t}\left\|X_{n}(s)\right\|_{H}^{2} d s \leq T C_{T}\left\|X_{0}\right\|_{H}^{2}
$$

for all $t \in[0, T]$ and all $n \geq 1$.

Consequently for $t>0$ there exists in $L^{2}(\Omega ; H)$ and in $L^{2}(\Omega \times[0, T] ; H)$ the process

$$
X(t):=\sum_{k=1}^{\infty} Y_{k}(t) h_{k}
$$

and the a priori estimates (3.11) and (3.12) hold also for $X(t)$.

Since $Y_{k}(0)=\left(X_{0}, h_{k}\right)$ holds, we obtain $X_{0}=\sum_{k=1}^{\infty}\left(Y_{k}(0), h_{k}\right) h_{k}$.

It holds for $X_{n}(t)$

$$
\begin{gathered}
X_{n}(t)=\sum_{k=1}^{n} \exp \left\{\lambda_{k} a t\right\}\left(X_{0}, h_{k}\right) h_{k} \\
+\sigma \int_{0}^{t} \sum_{k=1}^{n} \exp \left\{\lambda_{k} a(t-s)\right\} \mu_{1 k}\left(X_{n}(s), h_{k}\right) h_{k} d B_{k}^{h}(s)
\end{gathered}
$$




$$
+\eta \int_{0}^{t} \sum_{k=1}^{n} \exp \left\{\lambda_{k} a(t-s)\right\} \mu_{2 k}\left(X_{n}(s-), h_{k}\right) h_{k} d \tilde{\pi}_{k}(s) .
$$

Consequently, we get by the definition of the semigroup $\mathcal{T}_{t}$ and the operators $\Phi_{1}, \Phi_{2}$

$$
d X_{n}(t)=\mathcal{T}_{t} X_{n}(0)+\sigma \int_{0}^{t} \mathcal{T}_{t-s} \Phi_{1} X_{n}(s) d B^{h}(s)+\eta \int_{0}^{t} \mathcal{T}_{t-s} \Phi_{2} X_{n}(s-) d \tilde{\pi}(s),
$$

where $B^{h}$ and $\tilde{\pi}$ are the cylindrical fractional Brownian motion and the cylindrical centered Poisson process defined by the sequences $\left(B_{k}^{h}(t)\right)_{t \geq 0}, k \in\{1,2, \ldots\}$, and $\left(\tilde{\pi}_{j}(t)\right)_{t \geq 0}, j \in\{1,2, \ldots\}$.

With the definition of $X(t)$ from formula (3.13) and the a priori estimates (3.11), (3.12), with (3.14), by the definition of $Y_{k}(t)$ and the definitions of the stochastic integrals it is easy to prove, that the following result holds:

Theorem 3.4. The process $(X(t))_{t \in[0, T]}$ with $X(t)=\sum_{k=1}^{\infty} Y_{k}(t) h_{k}, t \in[0, T]$, solves

$$
X(t)=\mathcal{T}_{t} X_{0}+\sigma \int_{0}^{t} \mathcal{T}_{t-s} \Phi_{1} X(s) d B^{h}(s)+\eta \int_{0}^{t} \mathcal{T}_{t-s} \Phi_{2} X(s-) d \tilde{\pi}(s)
$$

for all $t \in[0, T]$.

Remark 3.5. The last theorem shows, that $(X(t))_{t \in[0, T]}$ is the mild solution of

$$
d X(t)=a A X(t) d t+\sigma \Phi_{1} X(t) d B^{h}(t)+\eta \Phi_{2} X(t-) d \tilde{\pi}(t), X(0)=X_{0} .
$$

\section{Parameter estimation}

In what follows we assume $Y_{k}(0)>0$ and $1+\eta \mu_{2 k}>0$ for all $k \in\{1,2, \ldots\}$. Let $k \in\{1,2, \ldots\}$ be arbitrary. We introduce a method to estimate the parameter $a$ in equation (3.2) for the process $\left(Y_{k}(t)\right)_{t \in[0, T]}$. By construction we can interpret this process as the process corresponding to the $k$-th Fourier coefficient of $(X(t))_{t \in[0, T]}$ with respect to $h_{k}$. We introduce the process

$$
\begin{aligned}
\xi_{k}(t):= & \ln \left(Y_{k}(t)\right)=\ln \left(Y_{k}(0)\right)+\sigma \mu_{1 k} B_{k}^{h}(t)-\frac{1}{2} \sigma^{2} \mu_{1 k}^{2} t^{2 h} \\
& +a \lambda_{k} t-\nu \eta \mu_{2 k} t+\ln \left(1+\eta \mu_{2 k}\right) \pi_{k}(t) .
\end{aligned}
$$

Then,

$$
\begin{aligned}
E\left(\xi_{k}(t)\right) & =E\left(\ln \left(Y_{k}(t)\right)\right)=\ln \left(Y_{k}(0)\right)-\frac{1}{2} \sigma^{2} \mu_{1 k}^{2} t^{2 h} \\
& +a \lambda_{k} t-\nu \eta \mu_{2 k} t+\left(1+\eta \mu_{2 k}\right) \nu t .
\end{aligned}
$$

Remark 4.1. Parameter estimation problems involving maximum likelihood methods for equations of type (4.1) without Poisson processes were considered in [1]. 
We consider for $n \in\{1,2, \ldots\}$ and for $\beta>1$, such that $n^{\beta} \in \mathbb{N}$, the partitions

$$
t_{1}=\frac{1}{n}<t_{2}=\frac{2}{n}<\ldots<t_{n^{\beta}}=\frac{n^{\beta}}{n}=: T\left(n^{\beta}\right) .
$$

Having statistical observations for $X(\cdot)$ in this time points, we can calculate $\xi_{k}\left(t_{1}\right), \ldots, \xi_{k}\left(T\left(n^{\beta}\right)\right)$.

We introduce the following estimation criterion analogous to the one given in [7]:

$$
\min \left\{\sum_{i, j=1}^{n^{\beta}} \operatorname{cov}\left(\xi_{k}\left(t_{i}\right), \xi_{k}\left(t_{j}\right)\right): a>0\right\} \text {. }
$$

Equation (4.3) is a quadratic function with respect to $a$. The factor in front of $a^{2}$ is given by the positive term $\lambda_{k}^{2}\left(\sum_{i=1}^{n^{\beta}} t_{i}\right)^{2}$. Consequently, there is a unique estimator $\hat{a}\left(n^{\beta}\right)$.

Theorem 4.2. For every $k \in\{1,2, \ldots\}$ the estimator $\hat{a}\left(n^{\beta}\right)=$

$$
-\frac{\sum_{i=1}^{n^{\beta}}\left(\ln \left(Y_{k}(0)\right)-\eta \mu_{2 k} \nu t_{i}+\ln \left(1+\eta \mu_{2 k}\right) \nu t_{i}-\frac{1}{2} \sigma^{2} \mu_{1 k}^{2} t_{i}^{2 h}-\ln \left(Y_{k}\left(t_{i}\right)\right)\right)}{\lambda_{k} \sum_{i=1}^{n^{\beta}} t_{i}}
$$

is unbiased and weakly consistent for the parameter a.

Proof. If we substitute $\ln \left(Y_{k}\left(t_{i}\right)\right)$ in the right hand side of $\hat{a}\left(n^{\beta}\right)$, then we get

$$
\hat{a}\left(n^{\beta}\right)=a+\frac{\sum_{i=1}^{n^{\beta}}\left(\sigma B_{k}^{h}\left(t_{i}\right)+\ln \left(1+\eta \mu_{2 k}\right) \tilde{\pi}_{k}\left(t_{i}\right)\right)}{\lambda_{k} \sum_{i=1}^{n^{\beta}} t_{i}} .
$$

Consequently, the estimator is unbiased. We get

$$
\begin{aligned}
& E\left[\hat{a}\left(n^{\beta}\right)-a\right]^{2}=E\left[\frac{\sum_{i=1}^{n^{\beta}}\left(\sigma \mu_{1 k} B_{k}^{h}\left(t_{i}\right)+\ln \left(1+\eta \mu_{2 k}\right) \tilde{\pi}_{k}\left(t_{i}\right)\right)}{\lambda_{k} \sum_{i=1}^{n^{\beta}} t_{i}}\right]^{2} \\
& =\frac{\sum_{i, j=1}^{n^{\beta}}\left(\frac{\sigma^{2} \mu_{1 k}^{2}}{2}\left(t_{i}^{2 h}+t_{j}^{2 h}-\left|t_{i}-t_{j}\right|^{2 h}\right)+\left(\ln \left(1+\eta \mu_{2 k}\right)\right)^{2} \nu \min \left\{t_{i}, t_{j}\right\}\right)}{\lambda_{k}^{2} \sum_{i, j=1}^{n^{\beta}} t_{i} t_{j}} .
\end{aligned}
$$


Further we have

$$
\begin{aligned}
\sum_{i, j=1}^{n^{\beta}} \frac{\sigma^{2} \mu_{1 k}^{2}}{2}\left(t_{i}^{2 h}+t_{j}^{2 h}-\left|t_{i}-t_{j}\right|^{2 h}\right) \leq \sum_{i, j=1}^{n^{\beta}} \frac{\sigma^{2} \mu_{1 k}^{2}}{2}\left(t_{i}^{2 h}+t_{j}^{2 H}\right) \\
\leq \sigma^{2} \mu_{1 k}^{2} \sum_{i, j=1}^{n^{\beta}}\left(\frac{n^{\beta}}{n}\right)^{2 h}=\sigma^{2} \mu_{1 k}^{2} \frac{n^{\beta(2+2 h)}}{n^{2 h}}, \\
\sum_{i, j=1}^{n^{\beta}}\left(\ln \left(1+\eta \mu_{2 k}\right)\right)^{2} \nu \min \left\{t_{i}, t_{j}\right\} \leq(\ln (1+\eta \mu 2 k))^{2} \nu \sum_{i, j=1}^{n^{\beta}}\left(\frac{n^{\beta}}{n}\right) \\
=\left(\ln \left(1+\eta \mu_{2 k}\right)\right)^{2} \nu \frac{n^{3 \beta}}{n}
\end{aligned}
$$

and

$$
\sum_{i, j=1}^{n^{\beta}} t_{i} t_{j}=\frac{1}{n^{2}}\left(\sum_{i=1}^{n^{\beta}} i\right)^{2}=\frac{n^{2 \beta}\left(n^{\beta}+1\right)^{2}}{4 n^{2}} \geq \frac{n^{4 \beta}}{4 n^{2}}
$$

Consequently,

$$
\begin{aligned}
& \lim _{n \rightarrow \infty} E\left[\hat{a}\left(n^{\beta}\right)-a\right]^{2} \\
& \leq \lim _{n \rightarrow \infty} \frac{4}{\lambda_{k}^{2}}\left(\sigma^{2} \mu_{1 k}^{2} \cdot n^{(2-2 h)+\beta(2+2 h-4)}+\left(\ln \left(1+\eta \mu_{2 k}\right)\right)^{2} \nu \cdot n^{(2-1)+\beta(3-4)}\right) \\
& \leq \lim _{n \rightarrow \infty} \frac{4}{\lambda_{k}^{2}}\left(\sigma^{2} \mu_{1 k}^{2} \cdot n^{(2-2 h)-\beta(2-2 h)}+\left(\ln \left(1+\eta \mu_{2 k}\right)\right)^{2} \nu \cdot n^{1-\beta}\right)=0
\end{aligned}
$$

and the weak consistency follows for $\beta>1$.

Remark 4.3. 1. In a similar manner we can calculate estimates for $\sigma^{2}$ and $\nu$. Then, we need the condition $\beta>4 h-1$ to prove the weak consistency.

2. The estimation of $\eta$ is difficult. A possibility consists in the application of an approximation of $\tilde{\pi}_{k}$ by Brownian motions $\left(B_{k}\right)_{k \geq 1}$ with $E\left(B_{k}^{2}(t)\right)=\nu t$ by using the Central Limit Theorem.

3. If $\eta=0$, then the random variable

$$
\frac{\lambda_{k}^{2}}{4} \cdot \frac{\hat{a}\left(n^{\beta}\right)-a}{\sigma^{2} \mu_{1 k}^{2} \cdot n^{(2-2 h)-\beta(2-2 h)}}
$$

is asymptotically $N(0,1)$ distributed.

Moreover, we prove the following result:

Theorem 4.4. Consider $\beta>\frac{3-2 h}{2-2 h}$ such that $n^{\beta} \in \mathbb{N}$. Then the estimate (4.4) for the parameter $a$ is strongly consistent for all $k \in\{1,2, \ldots\}$.

Proof. It is well known that, if for all $\varepsilon>0$ the relation

$$
\sum_{n=1}^{\infty} P\left(\left|\hat{a}\left(n^{\beta}\right)-a\right|>\varepsilon\right)<\infty
$$


holds, then $\lim _{n \rightarrow \infty} \hat{a}\left(n^{\beta}\right)=a$ with probability 1 .

Let $k \in\{1,2, \ldots\}$ be arbitrary. We know from the end of the proof of the last theorem that for the variance of $\hat{a}\left(n^{\beta}\right)$ we can write

$$
V\left(\hat{a}\left(n^{\beta}\right)\right) \leq \frac{4}{\lambda_{k}^{2}}\left(\sigma^{2} \mu_{1 k}^{2} \cdot n^{(2-2 h)-\beta(2-2 h)}+\left(\ln \left(1+\eta \mu_{2 k}\right)\right)^{2} \nu \cdot n^{1-\beta}\right) .
$$

Then, by using Chebyshev's inequality we obtain

$$
\begin{aligned}
& \sum_{n=1}^{\infty} P\left(\left|\hat{a}\left(n^{\beta}\right)-a\right|>\varepsilon\right) \leq \sum_{n=1}^{\infty} \frac{1}{\varepsilon^{2}} V\left(\hat{a}\left(n^{\beta}\right)\right) \\
& \leq \frac{4}{\lambda_{k}^{2}} \sum_{n=1}^{\infty} \sigma^{2} \mu_{1 k}^{2} \cdot n^{(2-2 h)-\beta(2-2 h)}+\frac{4}{\lambda_{k}^{2}} \sum_{n=1}^{\infty}\left(\ln \left(1+\eta \mu_{2 k}\right)\right)^{2} \nu \cdot n^{1-\beta} .
\end{aligned}
$$

Obviously, the first, respectively, the second sum on the right hand side of the last inequality are convergent, if

$$
\beta>\frac{3-2 h}{2-2 h}, \text { respectively, } \beta>2 .
$$

Hence, we get the statement for $\beta>\frac{3-2 h}{2-2 h}>2($ since $1 / 2<h<1)$.

\section{References}

[1] Bertin, K., Torres, S., Tudor, C. A., Maximum-likelihood estimators and random walks in long memory models, Statistics, 45(2011), no. 4, 361-374.

[2] Cialenco, I., Parameter estimation for SPDEs with multiplicative fractional noise, Stoch. Dyn., 10(2010), no. 4, 561-576.

[3] Gichman, I.I., Skorochod, A.W., Stochastische Differentialgleichungen, AkademieVerlag, 1971.

[4] Kozachenko, Y., Melnikov, A., Mishura, Y., On drift parameter estimation in models with fractional Brownian motion, Statistics, 49(2015), no. 1, 35-62.

[5] Long, H., Parameter estimation for a class of stochastic differential equations driven by small stable noises from discrete observations, Acta Math. Sci. Ser. B (Engl. Ed.), 30(2010), no. 3, 645-663.

[6] Lototsky, S.V., Rozovsky, B.L., Stochastic partial differential equations, Springer, 2017.

[7] Lueddeckens, J., Fraktale stochastische Integralgleichungen im White-Noise-Kalkül, Dissertation Martin-Luther-Universität Halle-Wittenberg, April 2017.

[8] Maslowski, B., Tudor, C.A., Drift parameter estimation for infinite-dimensional fractional Ornstein-Uhlenbeck process, Bull. Sci. Math., 137(2013), no. 7, 880-901.

[9] Nualart, D., Stochastic calculus with respect to fractional Brownian motion, Ann. Fac. Sci. Toulouse Math. (6), 15(2006), no. 1, 63-78.

[10] Protter, Ph., Stochastic Integration and Differential Equations, Springer, 1990.

[11] Xiao, W., Zhang, W., Zhang, X., Parameter identification for discretely observed geometric fractional Brownian motion, J. Stat. Comput. Simul., 85(2015), no. 2, 269-283. 
Wilfried Grecksch

Martin-Luther-University Halle-Wittenberg

Faculty of Natural Sciences II, Institute of Mathematics

06099 Halle (Saale), Germany

e-mail: wilfried.grecksch@mathematik.uni-halle.de

Hannelore Lisei

Babeş-Bolyai University

Faculty of Mathematics and Computer Sciences

1, Kogălniceanu Street

400084 Cluj-Napoca, Romania

e-mail: hanne@math.ubbcluj.ro

Jens Lueddeckens

Martin-Luther-University Halle-Wittenberg

Faculty of Natural Sciences II, Institute of Mathematics

06099 Halle (Saale), Germany

e-mail: jlueddeckens@web.de 